\title{
Effect of treadmill exercise on social interaction and tyrosine hydroxylase expression in the attention-deficit/ hyperactivity disorder rats
}

\author{
Dae-Jung Baek, Chae-Bin Lee, Seung-Soo Baek* \\ Department of Sport \& Health Science, College of Natural Science, Sangmyung University, Seoul, Korea
}

Attention-deficit/hyperactivity disorder (ADHD) involves clinically heterogeneous dysfunctions of sustained attention, with behavioral hyperactivity and impulsivity. The exact underlying mechanisms of ADHD are not known, however, impairment of dopaminergic system in the nigrostriatal pathway was suggested as the one of the possible mechanisms of ADHD. Tyrosine hydroxylase (TH) is the rate-limiting enzyme that is involved in the synthesis of dopamine. Spontaneous hypertensive rats have been used as the animal model for ADHD. Physical exercise is known to restore the brain functions disrupted by several neurodegenerative and psychiatric disorders. In the present study, we investigated whether treadmill exercise exerts therapeutic effect on ADHD. Social interaction test for the evaluation of impulsivity was performed using spontaneous hypertensive rats. TH expressions in the substantia nigra and striatum were evaluated by immunohistochemistry. In the present results, the rats of ADHD model showed hyper-social behaviors. TH expressions in the substantia nigra and striatum were decreased in the rats of ADHD model. Treadmill exercise alleviated hyper-social behaviors in the ADHD rats. TH expressions of ADHD rats were also enhanced by treadmill exercise. Here in this study, we showed that treadmill exercise effectively alleviates the ADHD-induced symptoms through enhancing of $\mathrm{TH}$ expression in the brain.

Keywords: Attention-deficit/hyperactivity disorder, Social interaction, Tyrosine hydroxylase, Substantia nigra, Striatum, Treadmill exercise

\section{INTRODUCTION}

Attention deficit/hyperactivity disorder (ADHD) is a developmental disorder, most often diagnosed in childhood (Pastor and Reuben 2008) and characterized by attention deficit, hyperactivity, and impulsivity. In addition, ADHD rats often exhibit hyper-social behavior and hyper-responsiveness behavior (Dela Peña et al., 2013).

It has been widely known that dopamine plays a major role for the modulation of neuroendocrine functions, cognition, attention, reward, and behaviors including motor activity. Dysfunction of dopamine signaling has a relation to the brain disorders such as Parkinson's disease and ADHD (Yoon et al., 2007). Some studies showed that dysfunction of dopamine signaling in the midbrain substantia nigra $(\mathrm{SN})$ is one of the most possible mechanisms of the behavioral symptoms of ADHD (Bowton et al., 2010; Vol-

kow et al., 2007). SN plays an important role in brain functions, particularly in movement, motor planning, reward seeking, learning, and addiction (Ji et al., 2014). The nigral dopaminergic input to the striatum via the nigrostriatal pathway is intimately linked with the striatum's function (Nicola et al., 2000). Tyrosine hydroxylase $(\mathrm{TH})$ is the rate-limiting enzyme synthesizing catecholamine neurotransmitters, and it converts L-tyrosine to L-dihydroxyphenylalanine (L-DOPA), which is the rate-limiting step in the dopamine synthesis. TH activity is decreased according to the loss of dopamine neurons in the SN (Haavik and Toska, 1998).

Exercise improves recovery from nigrostriatal dopamine injury and changes the dopaminergic neurotransmission in nigrostriatal system (O'Dell et al., 2007). Physical activity enhanced working speed and social behavioral problems, and diminished hyperactivity in ADHD children (Majorek et al., 2004). Treadmill exercise

\footnotetext{
${ }^{*}$ Corresponding author: : Seung-Soo Baek

Department of Sport \& Health Science, College of Natural Science, Sangmyung

University, 20 Hongjimun 2-gil, Jongno-gu, Seoul 110-743, Korea

Tel: +82-2-2287-5133, Fax: +82 -2-2287-0075, E-mail: ssoop@smu.ac.kr

Received: October 16, 2014 / Accepted: October 18, 2014
}

This is an Open Access article distributed under the terms of the Creative Commons Attribution Non-Commercial License (http://creativecommons.org/licenses/by-nc/3.0/) which permits unrestricted non-commercial use, distribution, and reproduction in any medium, provided the original work is properly cited. 
ameliorated symptoms of ADHD rats (Ji et al., 2014; Kim et al., 2011). Despite a variety of studies on the effect of treadmill exercise on various neuro-psychiatric disorders (Heo et al., 2014; Jee et al., 2008), there is no available data on the effects of treadmill exercise on the symptoms relief in the ADHD patients.

In the present study, we investigated the effect of treadmill exercise on the social interaction in relation with $\mathrm{TH}$ expression in the ADHD rats.

\section{MATERIALS AND METHODS}

\section{Animals and treatments}

Adult male spontaneously hypertensive rats (SHR) weighing $210 \pm 10 \mathrm{~g}$ were used as the ADHD animal model because the SHR displays the major symptoms of ADHD, such as inattention, hyperactivity, and impulsiveness (Sagvolden, 2000). Wistar-Kyoto (WKY) rats weighing 210 $\pm 10 \mathrm{~g}$ were used as the control in this study, according to the previous study (Hopkins., 2009). The rats were housed under controlled temperature $\left(20 \pm 2^{\circ} \mathrm{C}\right)$ and lighting conditions (07:00-19:00), with food and water made available ad libitum throughout the experiments. Animals were randomly divided into four groups ( $n=12$ in each group): control group, ADHD group, ADHD-treadmill exercise group, and ADHD-methylphenidate-treated group. All animal procedures were performed in accordance with the animal care guidelines of the National Institutes of Health $(\mathrm{NIH})$ and the Korean Academy of Medical Sciences.

\section{Treadmill exercise protocol}

The rats in the treadmill exercise groups were subjected to run on a treadmill for 30 min once a day, seven times a week, continued for 28 days. Exercise load for the exercise groups consisted of running at a speed of 2 meters $/ \mathrm{min}$ for the first $5 \mathrm{~min}$, at a speed of 5 meters/min for the next $5 \mathrm{~min}$, and then at a speed of 8 meters/ $\min$ for the last $20 \mathrm{~min}$, with the $0^{\circ}$ inclination. This intensity corresponded to the low-intensity treadmill exercise (\% maximal oxygen consumption) according to the rats in this age (Bedford et al., 1979).

\section{Social interaction test}

The social interaction (SI) test was conducted, according to the previous studies (Sams-Dodd, 1998; Calzavara et al., 2011). The test was performed in an open-field $(97 \mathrm{~cm}$ in diameter and 32.5 $\mathrm{cm}$ high, with an open top and a floor divided into 19 similar parts) under dim light (25 W) (Fig. 1). Pairs of unfamiliar rats receiving the same treatment were placed simultaneously into the apparatus approximately $80 \mathrm{~cm}$ apart. Social behavior and general motor activity parameters were scored live for $10 \mathrm{~min}$. Time spent in active (sniffing and following) or passive (when animals lie next to each other within a distance of $5 \mathrm{~cm}$ from skin to skin) SI was scored for each rat. The total SI time was quantified for each rat by finding the sum of the time spent engaged in active and passive social behaviors. Locomotor activity (the number of floor squares entered) and rearing frequency (the number of times each animal stood on its hind legs) were also quantified. In all experiment, the observers were blind to the treatment and strain of the rats

\section{Tissue preparation}

For brain tissue preparation, the animals were fully anesthetized with Zoletil $50^{\circledast}(10 \mathrm{mg} / \mathrm{kg}$, i.p.; Vibac Laboratories, Carros,

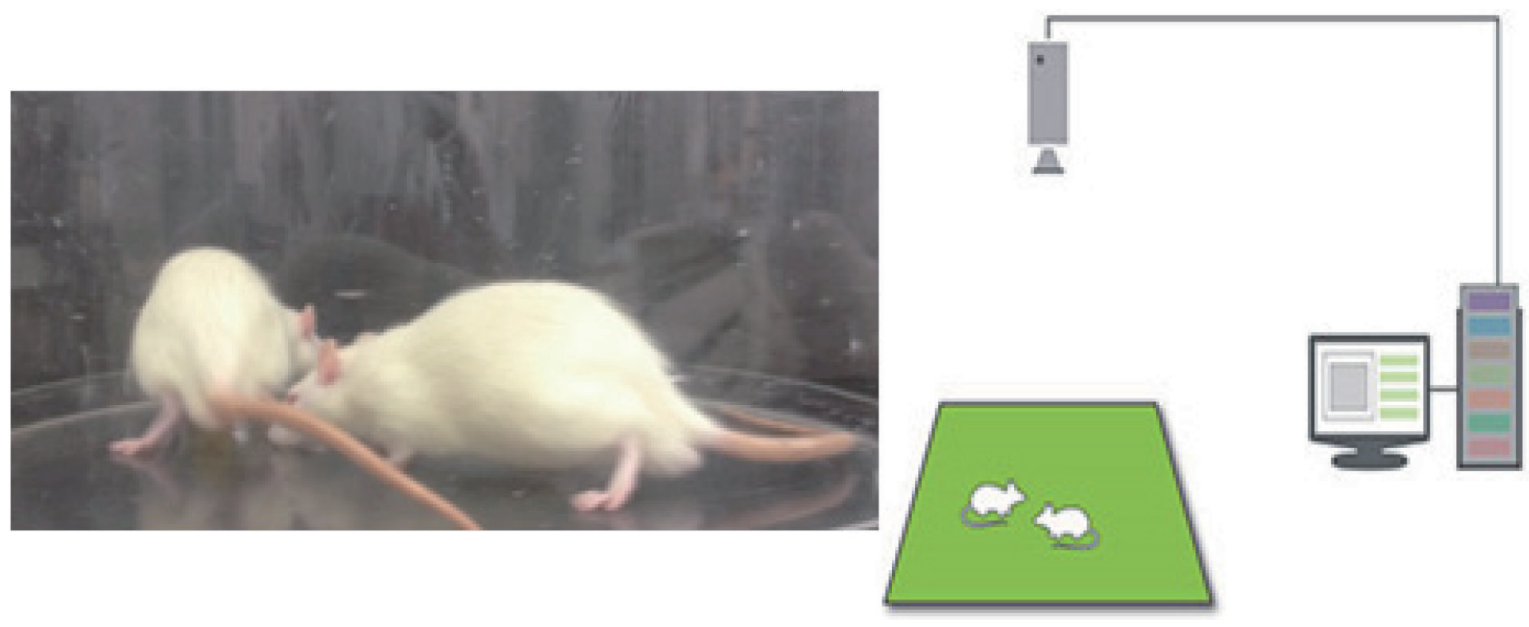

Fig. 1. Social interaction test. 
France), transcardially perfused with $50 \mathrm{mM}$ phosphate-buffered saline (PBS), and then fixed with a freshly prepared solution consisting of $4 \%$ paraformaldehyde (PFA) in $100 \mathrm{mM}$ phosphate buffer $(\mathrm{PB}, \mathrm{pH}$ 7.4). The brains were then removed, postfixed in the same fixative overnight, and transferred into a $30 \%$ sucrose solution for cryoprotection. Coronal sections of $40 \mu \mathrm{m}$ thickness were made using a freezing microtome (Leica, Nussloch, Germany).

\section{TH immunhistochemistry}

For immunolabeling of TH in the striatum and $\mathrm{SN}, \mathrm{TH}$ immunohistochemistry was performed as the previously described method (Kim et al., 2011). Free-floating tissue sections were incubated overnight with mouse anti-TH antibody (1:1,000, Santa Cruz Biotechnology, Santa Cruz, CA, USA) and the sections were then incubated for $1 \mathrm{~h}$ with biotinylated anti-mouse secondary antibody (1:200, Vector Laboratories, Burlingame, CA, USA). The sections were subsequently incubated with avidin-biotin-peroxidase complex (Vector Laboratories) for $1 \mathrm{~h}$ at room temperature. Immunoreactivity was visualized by incubating the sections in a solution consisting of $0.05 \%$ 3,3-diaminobenzidine (DAB) and $0.01 \%$ $\mathrm{H}_{2} \mathrm{O}_{2}$ in $50 \mathrm{mM}$ Tris-buffer (pH 7.6) for approximately $3 \mathrm{~min}$. The sections were then washed three times with PBS and mounted onto gelatine-coated slides. The slides were air-dried overnight at room temperature, and coverslips were mounted using Permount ${ }^{\circledR}$.

\section{Data analysis}

The area of striatum and SN from each slice was measured using Image-Pro® Plus computer-assisted image analysis system (Media Cyberbetics Inc., Silver Spring, MD, USA) attached to a light microscope (Olympus, Tokyo, Japan). The number of
TH-positive cells in the SN was counted hemilaterally through a light microscope (Olympus). TH-immunoreactive fiber density in the striatum was measured in $100 \mu \mathrm{m} \times 100 \mu \mathrm{m}$ square images of the striatum using animage analyzer (Multiscan, Fullerton, CA, USA). To estimate TH-staining density, optical densities were corrected for the non-specific background density, which was measured in the completely denervated parts of the striatum. TH-positive fiber density ratios in the striatum were calculated as follows: optical density in the lesion side/optical density in the intact side. The number of TH-positive cells in the $\mathrm{SN}$ was counted hemilaterally through a light microscope (Olympus, Tokyo, Japan).

Statistical analysis was performed using one-way ANOVA followed by Duncan's post-hoc test, and the results are expressed as the mean \pm standard error of the mean (SEM). Significance was set as $P<0.05$.

\section{RESULTS}

\section{Effect of treadmill exercise on social interaction in the ADHD-induced rats}

The activity score of the social interaction test is presented in Fig. 2. Non-aggressive behavior score was $11.14 \pm 1.21$ in the control group, $22.69 \pm 1.43$ in the ADHD group, $21.57 \pm 1.01$ in the ADHD-treadmill exercise group, and $20.61 \pm 0.78$ in the ADHD-methylphenidate-treated group. Aggressive behavior score was $3.89 \pm 0.53$ in the control group, $22.63 \pm 6.91$ in the ADHD group, $10.36 \pm 1.82$ in the ADHD-treadmill exercise group, and $6.48 \pm 0.51$ in the ADHD-methylphenidate-treated group. In the present results, non-aggressive and aggressive be-
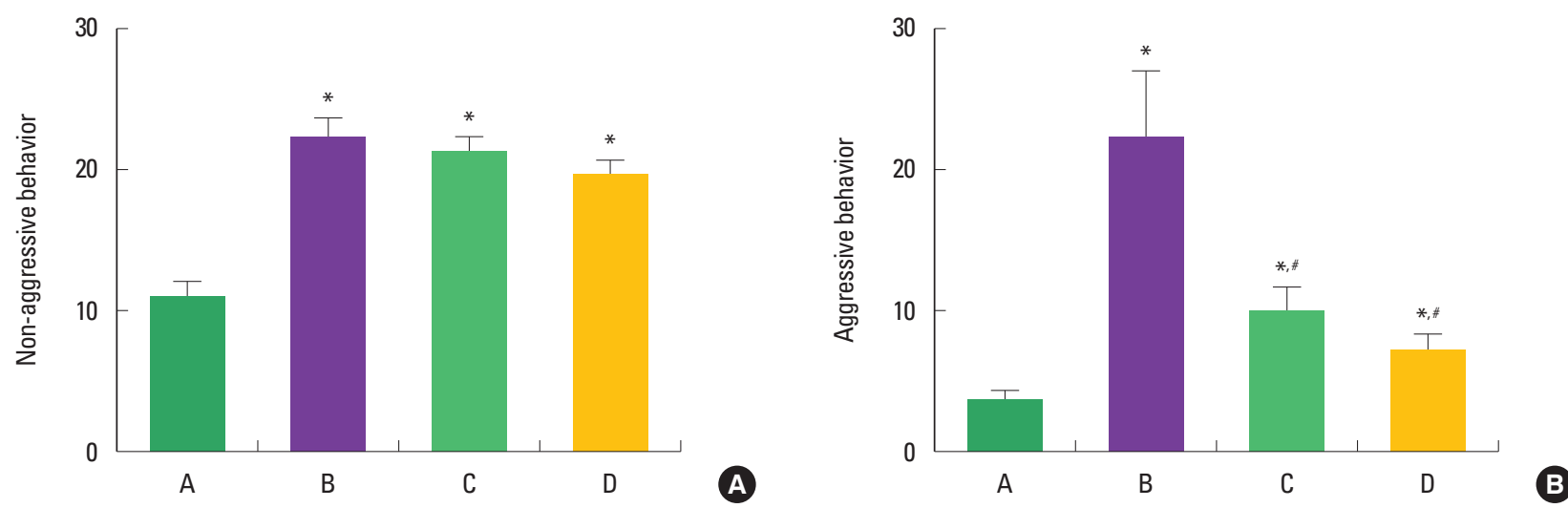

Fig. 2. Effect of treadmill exercise on social interaction in the attention-deficit hyperactivity disorder-induced rats. (A) No-aggressive behavior, (B) aggressive behavior A: Control group, B: ADHD group, C: ADHD-treadmill exercise group, D: ADHD-methylphenidate-treated group. * represents $P<0.05$ compared to the control group. " represents $P<0.05$ compared to the ADHD group. 
haviors in the ADHD rats were higher than that in the control rats. In contrast, both treadmill exercise and methylphenidate did not exert any effects on non-aggressive behaviors in the ADHD rats. Interestingly, aggressive behaviors were significantly decreased by treadmill exercise and methylphenidate treatment in the ADHD rats $(P<0.05)$.

Effect of treadmill exercise on TH expression in the SN of the attention-deficit/hyperactivity disorder-induced rats

Fig. 3 shows TH-positive cells in the SN of ADHD rats. The number of TH-positive cells in the $\mathrm{SN}$ was $438.16 \pm 35.21 / \mathrm{mm}^{2}$ in the control group, $245.98 \pm 37.45 / \mathrm{mm}^{2}$ in the ADHD group, $360.32 \pm 41.13 / \mathrm{mm}^{2}$ in the ADHD-treadmill exercise group, and
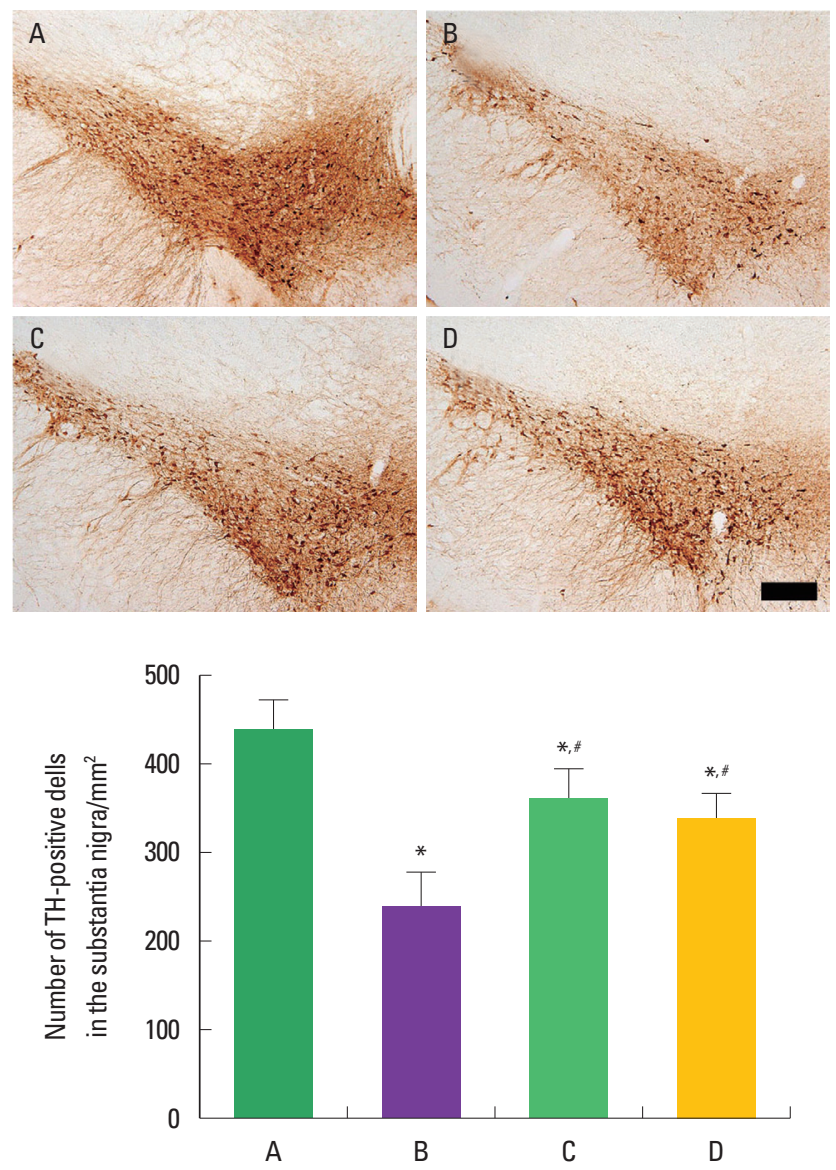

Fig. 3. Effect of treadmill exercise on tyrosine hydroxylase (TH) expression in the substantia nigra of the attention-deficit/hyperactivity disorder-induced rats. Upper: Photomicrographs of TH-positive cells in the substantia nigra, The scale bar represents $200 \mu \mathrm{m}$. Lower: Number of TH-positive cells in the substantia nigra. The data present means \pm standard error of the mean. (A) Control group, (B) ADHD group, (C) ADHD-treadmill exercise group, (D) ADHD-methylphenidate-treated group. ${ }^{*}$ represents $P<0.05$ compared to the control group. ${ }^{*}$ represents $P<0.05$ compared to the $\mathrm{ADHD}$ group.
$337.89 \pm 31.89 / \mathrm{mm}^{2}$ in the ADHD-methylphenidate-treated group. The results indicate that ADHD rats showed suppressed TH-positive cells in the $\mathrm{SN}$ compared to the control rats $(P<0.05)$. In contrast, the number of TH-positive cells in the $\mathrm{SN}$ was significantly enhanced by treadmill exercise and methylphenidate treatment in the ADHD rats $(P<0.05)$.

Effect of treadmill exercise on TH-immunoreactive fiber density in the striatum of the ADHD-induced rats

Fig. 4 shows TH-immunoreactive fiber density in the striatum of ADHD rats. The TH-immunoreactive fiber density in the striatum was $123.65 \pm 3.21 / \mathrm{mm}^{2}$ in the control group, $107.87 \pm 2.12$ / $\mathrm{mm}^{2}$ in the ADHD group, $119.25 \pm 1.96 / \mathrm{mm}^{2}$ in the ADHD-
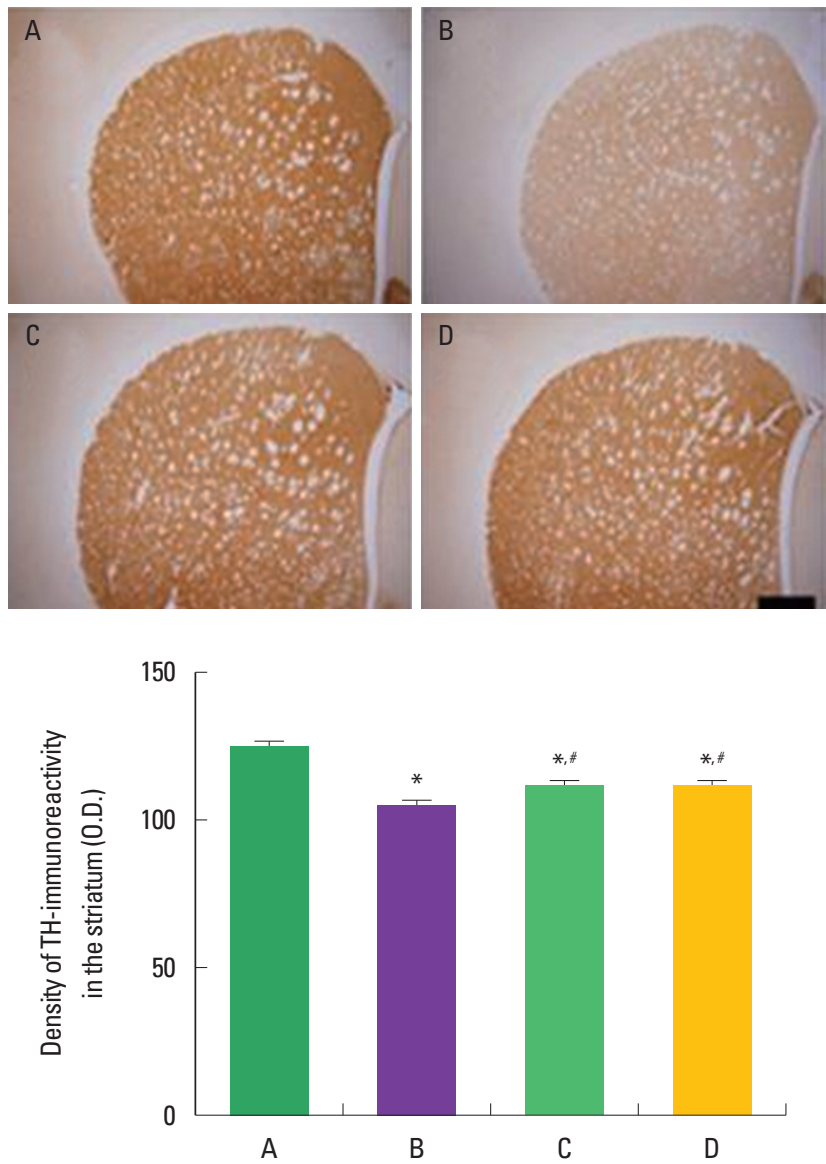

Fig. 4. Effect of treadmill exercise on tyrosine hydroxylase (TH)-immunoreactive fibers in the striatum of the attention-deficit/hyperactivity disorder-induced rats. Upper: Photomicrographs of TH-positive fibers in the striatum, The scale bar represents $200 \mu \mathrm{m}$. Lower: Optical density of TH-immunoreactive fibers in the striatum. The data present means \pm standard error of the mean. $(A)$ Control group, (B) ADHD group, (C) ADHD-treadmill exercise group, (D) ADHD-methylphenidate-treated group. *represents $P<0.05$ compared to the control group. "represents $P<0.05$ compared to the $\mathrm{ADHD}$ group. 
treadmill exercise group, and $120.03 \pm 2.23 / \mathrm{mm}^{2}$ in the ADHDmethylphenidate-treated group. The results indicate that the ADHD rats showed suppressed TH-immunoreactive fiber density in the striatum compared to the control rats $(P<0.05)$. In contrast, TH-immunoreactive fiber density in the striatum was significantly enhanced by treadmill exercise and methylphenidate treatment in the ADHD rats $(P<0.05)$.

\section{DISCUSSION}

SHRs were used as the most commonly accepted animal model for ADHD, because they exhibit hyperactivity, impulsivity, and poorly sustained attention (Sagvolden, 2000). This SHRs displayed non-aggressive and aggressive behaviors in the social interaction test as we expected. WKY rats, used as the control group, did not show any behavior symptoms of ADHD. These symptoms of ADHD, observed in the present study, are thought to be closely associated with hyper-social behavior and dysfunction of dopaminergic system in some brain regions compared with WKY rats.

Dopamine is a prominent neurotransmitter in regulating brain processes related with exercise. Hypo-function of the dopaminergic system leads to movement disorders (Servan-Schreiber et al., 1998). These conditions led to delayed aversion, development of hyperactivity in novel situations, impulsiveness, deficient attention, and increased behavioral variability (Sagvolden et al., 2005). Suppressed dopamine activity in caudate is associated with inattention in the adults with ADHD (Volkow et al., 2007). Many studies have suggested the possibility that dysfunction of dopamine signaling in the midbrain is one of the main mechanisms of hyperactivity (Bowton et al., 2010; Ji et al., 2014; Kim et al., 2011). In the present results, hyper-social behavior was observed in the ADHD rats, and expression of TH in the $\mathrm{SN}$ and striatum were significantly decreased in the ADHD rats. The present study suggests that hyper-social behavior in the ADHD rats is associated with down-regulation of dopamine in the $\mathrm{SN}$ and striatum.

Exercise is known to improve working speed and social behavior problems and to diminish hyperactivity in ADHD children (Majorek et al., 2004). Treadmill exercise showed alleviating effect on the hyperactivity in the ADHD rats (Hopkins et al., 2009; Kim et al. 2011). Increased dopamine synthesis by exercise enhanced the survival of dopaminergic neurons in the SN and treadmill running also alleviated some symptoms of ADHD (Hattori et al., 1994; Kim et al., 2011). In the present results, treadmill exercise suppressed hyper-social behavior and also enhanced TH expression in the $\mathrm{SN}$ and striatum.
In conclusion, the present study has shown that treadmill exercise has anxiolytic effect on social behavior in the ADHD with increasing effect of $\mathrm{TH}$ expression in the $\mathrm{SN}$ and striatum. Based on the present results, treadmill exercise may be a potential useful therapeutic strategy for the treatment of ADHD.

\section{CONFLICT OF INTEREST}

No potential conflict of interest relevant to this article was reported.

\section{ACKNOWLEDGMENTS}

This work was supported by 2012 Sangmyung University Research Foundation of Korea.

\section{REFERENCES}

Bedford TG, Tipton CM, Wilson NC, Oppliger RA, Gisolfi CV. Maximum oxygen consumption of rats and its changes with various experimental procedures. J Appl Physiol 1979;47:1278-1283.

Bowton E, Saunders C, Erreger K, Sakrikar D, Matthies HJ, Sen N, Jessen T, Colbran RJ, Caron MG, Javitch JA, Blakely RD, Galli A. Dysregulation of dopamine transporters via dopamine D2 autoreceptors triggers anomalous dopamine efflux associated with attention-deficit hyperactivity disorder. J Neurosci 2010;30:6048-6057.

Calzavara MB, Levin R, Medrano WA, Almeida V, Sampaio AP, Barone LC, Frussa-Filho R, Abílio VC. Effects of antipsychotics and amphetamine on social behaviors in spontaneously hypertensive rats. Behav Brain Res 2011;225:15-22.

Dela Peña I, Kim BN, Han DH, Kim Y, Cheong JH. Abuse and dependence liability analysis of methylphenidate in the spontaneously hypertensive rat model of attention-deficit/hyperactivity disorder (ADHD): what have we learned? Arch Pharm Res 2013;36:400-410.

Haavik J, Toska K. Tyrosine hydroxylase and Parkinson's disease. Mol Neurobiol 1998;16:285-309.

Hattori S, Naoi M, Nishino H. Striatal dopamine turnover during treadmill running in the rat: relation to the speed of running. Brain Res Bull 1994;35:41-49.

Heo YM, Shin MS, Lee JM, Kim CJ, Baek SB, Kim KH, Baek SS. Treadmill exercise ameliorates short-term memory disturbance in scopolamine-induced amnesia rats. Int Neurourol J 2014;18:16-22.

Hopkins ME, Sharma M, Evans GC, Bucci DJ. Voluntary physical exercise alters attentional orienting and social behavior in a rat model of 
attention-deficit/hyperactivity disorder. Behav Neurosci 2009;123:599606.

Jee YS, Ko IG, Sung YH, Lee JW, Kim YS, Kim SE, Kim BK, Seo JH, Shin MS, Lee HH, Cho HJ, Kim CJ. Effects of treadmill exercise on memory and c-Fos expression in the hippocampus of the rats with intracerebroventricular injection of streptozotocin. Neurosci Lett 2008;443:188192.

Ji ES, Kim CJ, Park JH, Bahn GH, Duration-dependence of the effect of treadmill exercise on hyperactivity in attention deficit hyperactivity disorder rats. J Exerc Rehabil 2014; 10:75-80.

Kim H, Heo HI, Kim DH, Ko IG, Lee SS, Kim SE, Kim BK, Kim TW, Ji ES, Kim JD, Shin MS, Choi YW, Kim CJ. Treadmill exercise and methylphenidate ameliorate symptoms of attention deficit/hyperactivity disorder through enhancing dopamine synthesis and brain-derived neurotrophic factor expression in spontaneous hypertensive rats. Neurosci Lett 2011;504:35-39.

Majorek M, Tüchelmann T, Heusser P. Therapeutic Eurythmy-movement therapy for children with attention deficit hyperactivity disorder (ADHD): a pilot study, Complement Ther Nurs Midwifery 2004;10: 46-53.

Nicola SM, Surmeier J, Malenka RC. Dopaminergic modulation of neuronal excitability in the striatum and nucleus accumbens. Annu Rev Neurosci 2000;23:185-215.

O'Dell SJ, Gross NB, Fricks AN, Casiano BD, Nguyen TB, Marshall JF.
Running wheel exercise enhances recovery from nigrostriatal dopamine injury without inducing neuroprotection. Neuroscience 2007; 144:1141-1151.

Pastor PN, Reuben CA. Diagnosed attention-deficit hyperactivity disorder and learning disability: United States, 2004-2006. Vital Health Stat 2008;10:1-14.

Sagvolden T. Behavioral validation of the spontaneously hypertensive rat (SHR) as an animal model of attention-deficit/hyperactivity disorder (AD/HD). Neurosci Biobehav Rev 2000;24:31-39.

Sagvolden T, Russell VA, Aase H, Johansen EB, Farshbaf M. Rodent models of attention-deficit/hyperactivity disorder. Biol Psychiatry 2005;57:1239-1247.

Sams-Dodd F. Effects of dopamine agonists and antagonists on PCP-induced stereotyped behaviour and social isolation in the rat social interaction test. Psychopharmacology, 1998;135:182-193.

Volkow ND, Wang GJ, Newcorn J, Telang F, Solanto MV, Fowler JS, Logan J, Ma Y, Schulz K, Pradhan K, Wong C, Swanson JM. Depressed dopamine activity in caudate and preliminary evidence of limbic involvement in adults with attention-deficit/hyperactivity disorder. Arch Gen Psychiatry 2007;64:932-940.

Yoon MC, Shin MS, Kim TS, Kim BK, Ko IG, Sung YH, Kim SE, Lee HH, Kim YP, Kim CJ. Treadmill exercise suppresses nigrostriatal dopaminergic neuronal loss in 6-hydroxydopamine-induced Parkinson's rats. Neurosci Lett 2007;423:12-17. 